\title{
Modelos de inferência causal: análise crítica da utilização da estatística na epidemiologia
}

\author{
Models of causal inference: advances in and the obstacles to the growing use \\ of statistics in epidemiology
}

Dina Czeresnia, Maria de Fútima Militão de Albuquerque

Departamento de Epidemiologia da Escola Nacional de Saúde Pública da Fundação Oswaldo Cruz (FIOCRUZ) - Rio de Janeiro, $B J$ - Brasil (D. C.),

Núcleo de Estudos em Saúde Coletiva/CPQAM - Fundaçao Oswaldo Cruz (FIOCRUZ) - Departamento de Medicina

Clinica/Centro de Ciências da Saúde da Universidade Federal de Pemambuco - Recife, PE - Brasil (M.F.M.A.)

\begin{abstract}
Discute-se a base de construçăo do conceito de risco, a partir da descriçăo do modelo de inferéncia causal de Fubin, desenvolvido no ambito de estatística aplicade, $\theta$ incorporado por uma vertente da epidemiologia. A apresentação das premissas da inferência causal torna visivel as passagens lógicas assumidas na construçăo do concelto de risco, permitindo entendè-io "por dentro". Esta vertente tenta demonstrar que a estatística ́ capaz de ínferir causalidade ao invés de simplesmente evidenciar associaç̋es estatisticas, estimando em um modelo o que é deflnido como o efelto de uma causa. A partir desta distinçăo entre procedimentos de inferéncia causal e de associaçăo, busca-se distingulr também o que seria a dimensáo epidemiológica dos conceitos, em contrapartida a uma dimensăo simplesmente estatlstlca. Nesse contexto, a abordagem dos conceitos de interacáo e confusắo torna-se maís complexa. Busca-se apontar as reduçōes que se operam nas passagens da construçăo metodológica do risco. Tanto no contexto de inferências individuais, quanto populacionals, esta construçăo metodológica impóe IImltes que precisam ser considerados nas aplicaçठøes teóricas e práticas da epidemiologia.
\end{abstract}

Risco. Inferência. Causalidade. Modelos de risco proporcionais.

\section{Introdução}

Uma questảo considerada fundamental na epidemiologia é a conceituação e operacionalização metodológica da causalidade. Identificar causas é uma das maneiras do pensamento científico abordar a explicação das origens de um fenômeno. A causa seria um agente eficaz, e desvendá-la garantiria um conhecimento maior a respeito do fenômeno estudado, na medida em que é possível intervir sobre um efeito quando se remonta à sua causa.
A causalidade foi trabalhada na epidemiologia, a partir da busca da causa verdadeira e específica da doença. Essa abordagem ganhou legitimidade com a identificação de agentes específicos responsáveis pela transmissão de doenças infecciosas. No entanto, a partir da necessidade de contornar a "ignorância" a respeito dos processos causais das chamadas doenças não transmissíveis ou crônicodegenerativas, o tratamento conceitual e metodologico da causalidade deslocou-se para a quantificação do risco. A busca da identificação de fatores de risco numa rede de múltiplas causas

Separates/Reprints: Dina Czeresnia - Departamento de Epidemiologia de Escola Nacional de Saúde Pública da FIOCRUZ - Rua Leopoldo Buthóes, 1480 - $8^{\circ}$ andar - Manguinhos - 21041-210 - Rio de Janeiro - RJ - Brasil Recebido em 27.10.1994. Aprovado em 30.5.1995. 
ampliou o desenvolvimento metodológico da epidemiologia ${ }^{25}$.

Esse processo vem ocorrendo através de uma crescente utilização de recursos estatísticos cada vez mais sofisticados. O conceito de risco e suas implicaçôes na epidemiologia têm sido estudados por vários autores ${ }^{1.5}$. As questões debatidas, em geral, são as reduções que se operam ao separar-se da realidade complexa e mutável, cadeias causais independentes quantificadas através de relações lineares.

O risco é uma medida de associação estatística, incapaz de inferir diretamente a causalidade. Bradford Hill citado por Rothman ${ }^{23}$, em 1965 já sugeriu alguns critérios para avaliar a natureza causal ou nâo de uma associação epidemiológica ${ }^{23}$. Demonstrar que as estimativas de risco poderiam evidenciar uma relação de causa e efeito fortalecia o caráter científico da pesquisa epidemiológica, respaldando eventuais medidas tomadas em resposta aos problemas de saúde pública. Entre os famosos critérios de Hill, o de "plausibilidade biológica" aponta para uma fragilidade importante do conceito de risco. Enn última instância, segundo esse criterrio, a biologia é que seria capaz de legitimar as associaçős estimadas nos estudos epidemiológicos.

Dessa forma, ao substituir a identificação da causa pela estimativa probabilística do risco, a epidemiologia teria construído sua identidade a partir de um conceito que não tem autonomia. Tendo como base de raciocínio que a construção da abordagem do risco foi decorrente da "ignorância" a respeito dos processos causais nas chamadas doenças crônicas, não se poderia afirmar que ocorreu "superação" do pensamento causal anterior.

$\mathrm{Na}$ verdade, o raciocínio epidemiológico ainda traz na sua essência a força do pensamento causal. Isso fica evidente nas investigaç̄̄es recentes acerca da etiologia da Imunodeficiência Adquirida (AIDS). As pesquisas estavam orientadas no sentido de encontrar a causa da imunodeficiência. Por analogia ao modelo da hepatite $\mathrm{B}$, afimnou-se a natureza transmissível da doença passando-se a buscar o agente causal. Durante esse processo, foi significativo como os estudos, que estimaram uma forte associação entre os casos de AIDS e o uso do "nitrito de amila", foram demonstrados como equivocados por não terem uma base biológica consistente ${ }^{26}$.

Ao mesmo tempo, tem-se reafirmado que o estudo de cofatores numa abordagem multicausal amplia a compreensāo das causas envolvidas na variabilidade da expressão das doenças. Os estudos epidemiológicos, realizados a partir de mode- los multifatoriais, relacionariam eventos clínicos $\mathrm{e}$ biológicos dentro de uma matriz de experiência social e de comportamento humano ${ }^{20}$, possibilitando uma abordagem mais complexa da etiologia das doenças.

Porém, seria necessário analisar a lógica de construção dos modelos de risco e como, através deles, é apreendido o significado das doenças e das formas sociais de lidar com elas. O desenvolvimento metodológico da epidemiologia, através do conceito de risco, tem contribuído decisivamente para a consolidação de práticas de intervenção sanitária que privilegiam apelos a mudanças comportamentais individuais. Os chamados fatores de risco (hábito de fumar, consumo de álcool, uso de drogas, alimentação deficiente, falta de exercícios, promiscuidade, entre outros) tendem a responsabilizar os indivíduos pelo surgimento de suas doenças, deslocando a ênfase de açōes coletivas de saúde.

O presente trabalho pretende discutir a base de construção do conceito de risco, a partir da apresentação do modelo de inferência causal de Rubin desenvolvido no âmbito da estatística aplicada ${ }^{13}$. A apresentação das premissas desse modelo torna visível as passagens lógicas assumidas na construção do conceito de risco, permitindo entendê-lo "por dentro". Esta vertente da epidemiologia tenta demonstrar que a estatística é capaz de inferir causalidade ao invés de simplesmente evidenciar associações estatísticas, estimando em um modelo o que é definido como o efeito de uma causa. A epidemiologia, através da estatística, ao incorporar em modelos teorias que expressam mecanismos de fenômenos biológicos, conquistaria maior grau de autonomia e cientificidade para a disciplina. Nesta perspectiva, a definição da identidade da epidemiologia ancora-se, fundamentalmente, na estatística e na biologia.

Pretende-se chamar aterção também, para o fato de que este processo de crescente utilização da estatística pelo método epidemiológico sofre a influência de mudanças que vêm ocorrendo no âmbito das ciências naturais, cujo estatuto de cientificidade é privilegiado por esta vertente da epidemiologia. Nesse sentido, a disscussảo da conceituaçāo de objetividade vem influenciando o núcleo da disciplina, também, através da estatística.

\section{As Premissas do Modelo de Inferéncia Casual}

As formulações básicas do modelo de inferência causal de Rubin (citado por Holian ${ }^{13}$ ), são: 
- O foco de atençāo da inferência causal deve se deslocar da busca de causas de efeitos para buscar encontrar os efeitos de causas.

- No raciocínio causal, falar que A causa B é relativo à outra causa que inclui a condição "não A". Isso implica comparar a exposição com a não-exposição à causa, ou, na linguagem da experimentação, o tratamento com o não-tratamento ou controle.

- Para a inferência causal é fundamental que cada unidade seja potencialmente exposta a qualquer uma das causas. Nesse sentido, é fundamental o modo como os indivíduos sâo alocados nos grupos de comparação. Além disto, esta concepção exclui os atributos pessoais como passíveis de serem causas.

- O papel do tempo é importante. A causa ocorre e os indivíduos (unidades) existem em um contexto temporal específico. Além disso, as medidas das características dos individuos, que compõem as variáveis estudadas, também devem ser feitas em tempos particulares.

- Inferir causalidade diz respeito a efeitos de causas em indivíduos (unidades) específicos. Os efeitos de causas ocorrem na singularidade. Isto implica o chamado "problema fundamental da inferência causal": é impossível observar simultaneamente o valor do tratamento e do não-tratamento ( $\mathrm{A}$ e "não A") na mesma unidade. A inferência causal deste modo seria impossível.

- Como solução, no caso em que as unidades são indivíduos, faz-se uso da estatística substituindo a impossibilidade de observar o efeito causal em um indivíduo específico pela possibilidade de estimar o "efeito causal médio" em uma população de indivíduos.

- Essa estratégia implica a necessidade de trabalhar com amostras da população. É necessário, portanto, garantir que todos os indivíduos da amostra sejam passíveis de serem expostos igualmente ao tratamento e ao não-tratamento. Faz-se isso através da alocaçăo dos indivíduos nos grupos de comparação de maneira aleatória.

Rubin (citado por Holland ${ }^{13}$ ) acrescenta como questão fundamental a ser considerada neste modelo a assunçāo do "valor estável de tratamento da unidade" (SUTVA). Isto significa que os indivíduos são independentes entre si. Assume-se a priori que o valor do resultado do tratamento para um indivfduo é independente do tratamento ou nāo dos outros indivíduos. Esta premissa garantiria que o efeito médio estimado a partir da amostra seja igual ao efeito médio calculado a partir da população.
Evidentemente, essa premissa nāo é plausível em todas as circunstâncias, porque ela restringe o grupo a uma soma dos indivíduos sem considerar suas relaçōes. Essa questão vem sendo trabalhada no contexto de estudos de eficácia vacinal em doenças transmissíveis, onde o conceito de imunidade de grupo orienta a necessidade de transpor esse limite do método's.

Esse é um dos desenvolvimentos que se viabilizam a partir desta apresentação das premissas da inferência causal, pois ela torna evidentes alguns dos seus limites. Entretanto, caberia chamar atenção para o fato de que as reduções decorrentes desses artifícios estatísticos não trazem problemas a serem considerados apenas no caso das doenças transmissíveis, como será comentado adiante.

Ponto fundamental dessa abordagem (que concebe o procedimento estatístico da inferência causal distinto da simples associação) é distinguir também, o que seria a dimensão epidemiológica dos conceitos, em contrapartida à uma dimensão simplesmente estatística. Tendo como base essa distinçāo, enfatiza-se a necessidade da apresentaçāo prévia de teorias e modelos, que expressem as características biológicas dos processos estudados. Esta visão provoca modificaçōes na conceituação e metodologia de avaliaçāo da "interaçāo" e "confusão".

Para que possamos entender as implicaçōes deste desenvolvimento metodológico, será necessário caracterizar os conceitos de interação e confusão.

\section{Interação entre Causas e "Confusão"}

O estudo da relaçāo causa e efeito em epidemiologia, como vimos, desenvolve-se a partir do objetivo de estimar uma medida de efeito (risco) entre exposição e doença. Nesse processo, a identificação de interação entre causas e de "confounding" ou confusão é considerada fundamental.

Caracteriza-se a existência de interação, dentro de um mesmo mecanismo causal, quando na presença de dois ou mais fatores de risco o efeito resultante é diferente do simples efeito combinado pelos efeitos individuais ${ }^{23}$. Em relação ao "confounding" ou confusão, pode-se defini-la como uma "mistura" de efeitos, isto é, como a superposiçāo do efeito de um fator de risco independente, sobre a relação estimada entre a exposição e o evento ${ }^{23}$.

A discussāo interna da epidemiologia a respeito da operacionalização desses dois conceitos é intensa. Qual a metodologia mais adequa- 
da para a sua avaliação no contexto dos estudos epidemiológicos?

No caso da análise da intteração, por exemplo, discute-se se é mais "apropriado" utilizar modelos aditivos ou multiplicativos. Nos modelos aditivos, a combinação dos efeitos individuais é feita pela soma das diferenças de riscos atribuíveis, enquanto que nos modelos multiplicativos a interação é avaliada pelo produto dos riscos relativos ou "odds ratio" 22

O modelo de análise é muitas vezes escolhido em função apenas da simplicidade e da conveniência estatística. Esse procedimento, sem referência aos mecanismos biológicos envolvidos, torna a definiçāo de interaçăo arbitrária e dependente do modelo escolhido. Portanto, a interação estatística expressaria apenas a interdependência entre fatores dentro dos limites de um dado modelo de risco ${ }^{23.24}$.

É nesse sentido que se ressalta que a pesquisa epidemiológica deveria ir além da modelagem estatística, priorizando a etapa explicativa da análise. O seu objetivo é a busca de explicações para as relaçōes observadas, seja no que se refere à presença de "confounding" na estrutura do estudo, seja em relação à causa a partir de um modelo biologico subjacente.

A interaçāo deve, portanto, ser vista como uma característica biológica do fenômeno estudado cujo mecanismo deve ser explicitado previamente à escolha de um modelo de análise estatística ${ }^{22}$.

Da mesma forma, esta questāo está colocada na discussāo do "confounding". Neste caso também seria necessário definir previamente quais as variáveis a serem controladas. Ser "confounder" não é uma característica inerente de qualquer variável, ocorrendo somente no contexto de um estudo particular. Uma variável đe confusão deveria ter as seguintes características: ser um fator de risco para a doença entre os não expostos; estar associada com a exposição na população em estudo; e não ser uma variável intermediária na sequiência da exposição para o evento ${ }^{15}$.

No contexto de estudos experimentais, a possibilidade de alocação aleatória dos indivíduos tem sido classicamente considerada uma estratégia eficaz de garantir a comparação entre grupos de tratamento, e assim prevenir a confusão. Foi, portanto, no contexto de estudos observacionais, onde a alocação aleatória dos indivíduos nos grupos de comparação é impossível, que se sentiu maior necessidade de desenvolver conceitos e estratégias para melhor abordar a confusāo $\mathrm{O}^{23}$.

De modo semelhante ao que foi observado em relação à interação, a abordagem do "confoun- ding" na epidemiologia tendeu, muitas vezes, a se restringir ao âmbito técnico da estatística. É neste sentido que Miettinem e Cook ${ }^{15}$ e Greenland e Robins ${ }^{8}$ criticam que a conceituação de "confounding" seja feita a partir de um julgamento realizado na etapa da análise dos dados.

Caso o controle da variável de confusão, por estratificação ou técnicas multivariadas, não mude a estimativa de efeito, isto é, quando a estimativa "bruta" é igual à estimativa "ajustada" por estratos, a medida de efeito é assumida como representativa, ou seja, não existe "confounding".

Este critério que se baseia na análise técnica dos dados pode levar a fajsas conclusōes. Porém, um outro tipo de conceituação considera a confusão como surgindo a partir de diferenças "inerentes" ao risco, entre a população de expostos e não expostos. Ou seja, essas diferenças existiriam mesmo se a exposição estivesse inteiramente ausente de ambas as populações"s.

Portanto, prevenir "confounding" seria garantír que grupos expostos e não expostos possam ser comparáveis ou permutáveis, isto é, na ausência de exposição a proporção de casos entre os expostos e não expostos seria a mesma ${ }^{8}$. Deste modo, esta garantia de que os grupos podem ser comparados ou permutados entre si é o que tornaria o efeito identificável, aproximando as condições do estudo dos fundamentos do modelo de inferência causal.

Esta mudança na conceituação do "confounding" reforça a idéia de que é importante ter uma teoria explicitada a respeito do fenômeno biológico estudado. Reforça, também, a necessidade de uma definição anterior de quais as variáveis importantes a serem consideradas no modelo. Esta mudança desloca também, a lógica da utilização da modelagem matemática na análise multivariada.

\section{A Escolha do Modelo de Análise. O Papel da Subjetividade}

Na etapa da análise dos dados, o desenvolvimento de técnicas de análise multivariada proporciona, através de modelos estatísticos, uma maneira de controlar o "confounding" e verificar a interação ${ }^{23}$.

Uma das maneiras de trabalhar a modelagem é considerar no modelo todas as variáveis potencialmente envolvidas e suas interaçōes. Neste caso, terfamos um modelo dito "saturado", que está de certo modo coerente com uma postura de ignorância prévia a respeito do fenômeno estudado. 
Delega-se. desta forma. à etapa de análise o papel de escolher quais delas são pertinentes. Neste processo perde-se em precisão e qualidade 'x.

Lm outro tipo de abordagem considera que o modelo estatístico deve tentar reproduzir o que ocorre na natureza, assumindo um conjunto de restrições. Assumir restriçōes implicaria optar por incluir no modelo apenas as variáveis consideradas importantes. Neste caso, ganhar-se-ia em precisão. mas poderia existir o risco de não se incluir alguma variável que pudesse provocar "confounding". Esta estratégia, de fato, exigiria assumir premissas detalhadas sobre processos que muitas vezes são pouco conhecidos "

Outro aspecto que esta visão ressalta é que a adequação à uma função matemática não é um objetivo em si mesmo. O processo de modelagem ao adequar dados pode apagar características essenciais do processo biológico em estudo?

Dessa forma, cabe ao investigador ter uma teoria prévia a respeito do processo biológico em estudo: avaliar qual função matemática tem melhor condiçāo de expressá-lo e assumir quais variáveis säo importantes no modelo. "Desde que o verdadeiro estado da natureza é desconhecido, a magnitude do viés não pode ser conhecida. Portanto. a escolha é subjetiva e sujeita a erro":".

Esse desenvolvimento parte da aproximação a uma concepção de estatística não convencional. $O$ conceito de probabilidade nesta perspectiva não parte da assunção de ignorância prévia da distribuição do fenômeno estudado. Quantifica-se uma crença anterior, utilizando-se uma distribuição de probabilidade subjetiva. Os dados são então usados para atualizar esta distribuição pela regra de Bayes ${ }^{1 \%}$.

A alternativa bayesiana, apesar de antiga, vem sendo retomada recentemente em função da ampliação dos recursos técnicos da computação eletrônica ${ }^{\perp}$. Além disso. ela expressa uma concepção de objetividade distinta daquela que a estatística padrão utiliza. De certa forma, esta retomada é um reflexo da crescente discussão que vem ocorrendo a respeito do conceito de objetividade, no âmbito das ciências naturais.

Esta perspectiva argumenta que obter dados objetivos de uma análise estatística requer "input" subjetivo. O reconhecimento da subjetividade inerente à interpretação de dados possibilita que novas evidências possam ser integradas ao conhecimento anterior:

Critica-se assim, o conceito de objetividade dos modelos de análise convencionais. Estes, na verdade. também dependem das intenções do investigador ao, por exemplo. optar por colher determinados dados e não outros. A subjetividade não explícita seria muito mais perigosa porque é apresentada como "neutra" e "objetiva".

A estatística bayesiana, portanto, torna relativo o valor de verdade do dado quantitativo, assumindo-o enquanto uma construção. $O$ dado, desta forma, já não é visto como uma mensuração do real, em que a objetividade e rigor são garantidos através da neutralidade $e$ isenção de valor do investigador.

Provavelmente. inspirado nesta concepção de probabilidade, é que Greenland" questiona a alocação aleatória dos indivíduos em grupos de comparação como a melhor forma de prevenir a confusão em estudos epidemiológicos experimentais. Cada indivíduo teria unia probabilidade própria de expor-se a um evento. independentemente da exposição em estudo. Portanto. os indivíduos nâo seriam igualmente informativos. A alocação aleatória não evitaria a possibilidade destas diferenças tornarem a estimativa do risco "sub" ou "super" dimensionada. Ela apenas tornaria esta estimativa estatisticamente não viesada".

Dessa forma, alocar grupos de forma aleatória só seria útil na prevenção de confusão no caso de variáveis não conhecidas. Se as variáveis são conhecidas e podem ser medidas, seria melhor considerar essas diferenças no estudo, num modelo de análise estatística bayesiano:

No âmbito de estudos observacionais. uma questão que se coloca, a partir desta concepção, é a crítica à propriedade da utilização de estatísticas baseadas na rejeição da hipótese nula e no valor de p. Inclusive é ressaltado que, muitas vezes, ao invés de trabalhar com modelos de análise impróprios. seria melhor interpretar os dados de modo não estatístico. Isto poderia ser feito, entre outras formas, valorizando-se estudos descritivos bem trabalhados através de gráficos e tabelas'.

Um desdobramento possivel deste desenvolvimento. e sem dúvida promissor, seria conseguir superar metodologicamente outros modos clássicos de controle de viés que pressupōem a neutralidade do investigador. Como é o caso dos estudos "duplo cegos" e utilização de placebos em estudos experimentais.

\section{Discussão}

No decorrer do presente texto descrevemos os desenvolvimentos metodológicos suscitados por uma vertente da epidemiologia moderna. que se 
fundamenta no modelo de inferência causal de Holland e Rubin. Este modelo explicita as suas premissas, com o objetivo de estimar uma medida de efeito - o risco - entre exposiçảo e doença. Por essa razão é que, dentro desta perspectiva, são privilegiados os conceitos de interaçăo e confusão. cuja abordagem vem tornando-se mais complexa na epidemiologia. Destacamos como esse processo vem sofrendo a influência de uma concepção de probabilidade que reconhece a subjetividade como elemento de construção de "rigor" científico.

Neste ponto do trabalho pretende-se retomar a discussāo sobre a operacionalização da lógica da inferência causal com o objetivo de identificar passagens internas do método e premissas assumidas que explicitam alguns problemas.

Ressalta-se. inicialmente, a afirmação de que para a inferência causal é importante contextualizar no tempo os individuos, as causas e a ocorrência de efeitos". Caberia indagar com qual conceito de tempo este modelo opera. No âmbito da discussão da inferência causal. Holland ${ }^{\prime:}$ implicitamente considera duas maneiras de pensar a temporalidade. Por exemplo. em condições de laboratório. trabalhando fenômenos físicos, seria possível supor o tempo como reversível. isto é. poder-se-ia repetir em laboratório as condiçōes iniciais de uma experiência.

A experiência anterior não interferiria nas subseqüentes. No caso dos fenômenos biológicos estudados pela epidemiologia. as condiçōes iniciais da experiência não retornam. O tempo é irreversivel e unidirecional.

Sendo assim. o que significaria contextualizar no tempo um estudo epidemiológico ? Nào implicaria também em trabalhar a dimensão do tempo enquanto história? Sem dúvida. mesmo no contexto da biologia. as transformaçōes evolutivas vão ao encontro à crença de invariabilidade, fixidez e universalidade dos seres vivos. Isto se torna mais flagrante no caso de populações humanas. que são mediadas pela linguagem e pela história. construindo singularidades individuais e sociais.

Dessa forma. o que seria construir uma medida de efeito (risco) contextualizada no tempo? Até que ponto, ao contrário, esta medida pretende expressar características universais do homem. abstraindo o movimento e a diversidade?

Essas indagaçōes remetem à necessidade de se pensar. como internamente à lógica da inferência causal é construída a medida de efeito (risco). O "problema fundamental da inferência causal". como já vimos. é apresentado como a impossibilidade de observar o efeito da exposição e nào exposição simultaneamente no mesmo indivíduo Como solução, calcula-se o "efeito causal médio", trabalhando-se com grupos de população que possam ser comparáveis.

O risco, segundo esta lógica, seria medida de efeito para inferência individual. $O$ grupo. nesse caso, é utilizado como forma de viabilizar operacionalmente a inferência individual. Sendo assim. caberia analisar a propriedade da estimativa do "efeito causal médio" para a inferência individual. $\mathrm{O}$ que se colocaria em questão é esta necessidade interna do método, ao contornar o "problema fundamental da causalidade" fazer primeiro uma passagem do nível individual para o do grupo. voltando em seguida para o individual.

$\mathrm{O}$ que acontece nestas passagens? Será que a condição da individualidade é preservada? O risco só corresponderia à singularidade se os indivíduos fossem homogêneos. Porém. mesmo a epidemiologia não opera com a suposição de homogenei. dade dos indivíduos. Ela busca, como artifício metodológico. a comparação dos grupos através de uma distribuição homogênea das heterogeneidades individuais. O risco então não seria um conceito passível de ser transposto à singularidade sem mediaçōes:

Esta construção metodológica é coerente com a visăo que distingue risco - enquanto medida do probabilidade individual de uma derivação deste. a razão de densidade de incidência - enquanto medida capaz de estimar a força de morbidade em populaçōes:1.:. A duplicidade de objetivos de medidas construídas a partir da mesma lógica causal, poderia ser vista como ambiguiidade. pois a epidemiologia define-se como o estudo de doenças em populaçōes.

Porém. a abordagem individual do risco deixa clara a sua intenção de servir como subsídio à prática clínica e avaliação da tecnologia médica. A dominância desse objetivo na prática da epidemiologia moderna tem relegado a abordagem populacional, ligada à tradiçăo da saúde pública"

Caberia analisar também as implicações da utilização do "efeito causal médio" como medida populacional. Neste caso, esbarra-se na premissa do modelo da inferência causal que assume a independência entre os indivíduos que pertencem ao grupo estudado (SUTVA), como já foi apresentado.

No caso das doenças infecciosas. assumir a premissa de independência é mais facilmente questionável. $O$ estudo dessas doenças utiliza conceitos como os de infeçăo, suscetibilidade e imunidade. As relações entre eles produzem o con- 
ceito de "imunidade de grupo", que-não pode ser reduzida à soma da imunidade dos indivíduos, e termina por determinar a dinâmica de transmissảo: ${ }^{: 13}$. Pode-se dizer que o conceito de transmissão preserva um conteúdo relacional que tende a ser desconectado na conceituação das doenças "não transmissíveis" ou "crônico-degenerativas".

Certamente esse é o motivo da assunção do SUTVA ser considerada mais apropriada no caso dos estudos em doenças crônicas. Mas, como dizer que questões consideradas fatores causais de doenças crônicas como fumo, alimentação, violência, agentes tóxicos são "não transmissíveis"? Será que esses não são problemas transmitidos através da relação dos homens entre si e com a natureza? Será que assumidos a partir da sua dimensão cultural, estas questões não devem ser trabalhadas numa perspectiva de "imunidade de grupo" ou "suscetibilidade de grupo"? Sem dúvida. na perspectiva do "SUTVA", a população não é vista com propriedades de organização que produzem consequiências para a situação de saúde dos individuos.

Quando a pergunta colocada é qual a causa da distribuição desigual de doenças entre populaçōes. e não qual a causa dos casos, a lógica da independência dos indivíduos não seria a mais adequada'. Sendo assim, a construção metodológica do risco, enquanto "efeito causal médio", tanto na estimativa de inferências individuais quanto populacionais, apresenta limites que precisam ser considerados no momento de sua aplicação.

Sem dúvida, o desenvolvimento do método impõe artifícios inevitáveis na perspectiva de viabilizar sua operacionalização, contornando questōes como o "problema fundamental da inferência causal". Corre-se o risco, no entanto, de que as reduções, inevitáveis do ponto de vista da lógica interna do método, serem esquecidas e absorvidas como verdade. A passagem lógica poderia apagar características fundamentais do fenômeno estudado. Desta forma, quando o método é trabalhado sem a compreensão do significado das reduçōes que opera, pode transformar um artifício operacional em artefato. $O$ método, se reificado. estreita as possibilidades de compreensão da realidade.

Cabe, então, colocar uma questão óbvia de ser feita. mas não tão óbvia de ser respondida na prática do processo de investigação. Para que problemas um método é adequado e para que problemas não é? Em relação ao conteúdo deste texto. poder-se indagar de forma mais específica: para que questôes é pertinente esta abordagem que busca avaliar o efeito da causa contra a "não-causa", isolando relaçôes (ou cadeias) causais independentes"?

Deslocando para uma dimensão mais prática da discussão, pode-se dizer que. essa tem sido uma maneira considerada apropriada de estudar o efeito de exposiçōes individuais. como avaliação da eficácia de medicamentos, procedimentos clínicos e vacinas. Neste contexto, caberia ressaltar que o aprimoramento metodológico descrito no presente trabalho tem muito a contribuir. Um exemplo seria a possibilidade de criação de desenhos de estudo que conseguissem superar a necessidade de controlar o "viés" através da suposição de neutralidade. Sem dúvida. conseguir superar as necessidades técnicas de alocação aleatória. duplo cego e utilização de placebos. sem perder o estatuto de cientificidade. seria um desafio de modo nenhum irrelevante. Principalmente. quando se reivindica concretamente que os testes da eficácia de drogas e vacinas possam ser realizados numa perspectiva de maior rigor ético it:

Por outro lado sabe-se que o desenvolvimento metodológico descrito. permanece aprisionado a uma concepção de causalidade linear. Que isola um aspecto que variá. enquanto supõe os outros constantes.

Os modelos dinâmicos em epidemiologia. ao considerarem as relaçōes entre indivíduos e grupos de indivíduos assim como as mudanças que ocorrem entre estes com a passagem do tempo. permitem abordar interações mais complexas ${ }^{17}$. Ao trabalharem com conceitos distintos, como "limiar". "ponto de equilíbrio", "fluxo". permitem uma outra aproximação à causalidade. No entanto. de novo retoma-se a questão de como abordar a passagem do tempo e interações complexas. em fenômenos de saúde em populaçōes humanas, sem trabalhar a dimensão do tempo enquanto história. A demarcação do campo disciplinar da epidemiologia, restrito à biologia e à estatística, sem dúvida. dificulta esta possibilidade.

Finalmente, uma reflexão a ser feita diz respeito a característica do pensamento científico de transformar a base de onde algo surge, na causa que o produz. E da visão de que a revelação da causa ocupa lugar maior na explicação de um fenômeno. Pois, "não só as aparências nunca revelam espontaneamente o que se encontra por trás delas. Nenhuma coisa. nenhum lado de uma coisa se mostra sem que ativamente oculte os demais"(Arendt").

Está é uma característica incontornável do processo de construção do conhecimento. Ao optar por revelar e explicar algo. inevitavelmente 
oculta-se um outro lado. Ao selecionar determinadas causas, temas, conceitos e métodos excluem-se outras. E as opçōes correspondem a interesses, valores e necessidades.

É necessário ressaltar, que anterior ao método está a teoria. E é intrínseco à teoria a natureza subjetiva do pensamento e da açāo, correspondendo à presença de interesses e necessidades humanas. $O$ conteúdo de verdade de uma teoria não está dado a priori, independente de um juízo de valor. E o método, enquanto conjunto de estratégias de investigaçāo e de técnicas de análise, não deve estar alienado da teoria, através de modelos formais que ganham vida própria ${ }^{6}$.

\section{Referências Bibliográficas}

1. ALMEIDA FILHO, N. A clínica e a epidemiologia. Salvador, Abrasco, 1992.

2. ARENDT, H. A vida do espírito, o pensar, o querer, o julgar. Rio de Janeiro, Relume Dumará, 1992.

3. BERGER, J, O. \& BERRY, D. A. Statistical analysis and the illusion of objectivity. Am. Scient., 76: $159-65,1988$.

4. BRESLOW, N. Biostatistics and bayes (with discussion). Stat. Sci., 5: 269-98, 1990.

5. GOLDBERG, M. Este obscuro objeto da epidemiologia. In: Costa, D. C. Epidemiologia, teoria e objeto. São Paulo Hucitec/Abrasco, 1990

6. GONÇAIVES, R, B, M. Contribuição à discussào sobre as relaçòes entre teoria, objeto e método em epidemiologia, In: Congresso Brasileiro de Epidemiologia, $1^{9}$. Campinas, 1990. Anaśs, São Paulo, Abrasco, 1990.

7. GREENLAND, S. Limitation of the logistic analysis of epidemiologic data. Am.J. Epidemiol. 110: $693-8,1979$

8. GREENLAND, S. \& ROBBINS, J. M. Identifiability, exchangeability, and epidemiologic confounding. Int. J. Epidemiol., 15:412-8, 1986.

9. GREENLAND, S. Randomization, statistics, and causal inference. Epidemiology, 1:421-9, 1990.

10. GREENLAND, S, \& ROBINS, J. M. Ecologic studies: biases, fallacies, and counterexamples. Am. J. Epidemiol., 139: 747-60, 1994.

11. HALLORAN, M. E, et al. Direct and indiret effects in vaccine efficacy and effectivenes. $A m . J$. Epidemiol., 133: 323 -31, 1991.

12. HOLLAND, P.W. Statistics and causal inference. J. Am. Stat. Assoc, 81 (396): 945-960, 1986.

13. HORTON, M. Bugs, dings and placebos. In: Carte, E, \& Watney, S, ed, Taking libertines: AIDS and control cultural politics. London, Serpents Tail, 1989.

14. KLEINBALM, D. et al. Epidemiologic researcb: principles and quantitative metbods. New York, Van Nostrand Reinhold, 1992
Compreender a base da construçāo teórica e metodologica de um campo de conhecimento, não se justifica apenas para evidenciar os limites que reduzem a dimensão de problemas. Mas para permitir pensar como resolver problemas devidamente formulados, radicalizando as possibilidades de integrar as metodologias que possam resolvêlos da melhor forma possível.

\section{Agradecimentos}

Ao Claudio Struchiner pela colaboração e leitura do texto.

15. MIETTINEN, O. S. \& COOK, E. F. Confounding essence and detection. Am. J. Epidemiol. 114:593-603, 1981

16. MORGENSTERN, $H$. et al, Measures of disease incidence used in epidemiologic research. Int. J. Epidemiol., 9:97-104, 1980.

17. NOKES, D. J. \& ANDERSON. The use of mathematical models in the epidemiological study of infections diseases and in the desigs of mass immunization programmes. Epidemiol. Infec., 101: 1-20, 1988.

18. OPPENHEIMER, G. M. Causes, cases, and cohorts: the role of epidemiology in the historical construction of AIDS. In: Fee, E. \& Fox, D. M. ed. AIDS; the making of chronic disease. Berleey, University of California Press, 1992.

19. ROBINS, J. M. \& GREENLAND, S. The role of model selection in causal inference from monexperimental. Am. J. Epidemiol., 123: 392-402, 1986.

20. ROSE, G. Individuos enfermos y poblaciones enfermas. In: Organizacion Panamericana de la Salud. El desafio de la epidemiologia. Washington, D. C., 1988, p. 909-9. (OPS . Publ. Cient., 505).

21. ROTHMANN, D. L. \& EDGAR, H. Scientific rigor and medical realities: placebo trials in cancer and AIDS research, In: Fee, E. \& Fox, D. M., ed. AIDS: the making of a cbronic disease. Berleey, University of California Press, 1992

22. ROTHMAN, K. J. et al. Concepts of interation. Am. J. Epidemiol., 112: 467-70, 1980.

23. ROTHMAN, K. J. Modem epidemiology. Little, Brown and Company, Boston, 1986.

24. SIEMIATYCKI, J, \& THOMAS, D. C. Biological models and statistical interactions: an example from multistage carcinogenesis. Int. J. Epidemiol., 10: 383-7, 1981.

25. SUSSER, M. Epidemiology in the United States after World War. II. The evolution of technique. Epidemiol. Rev., 17:147-77, 1985. 
26. VANDENBROLCKE, J. P. \& PARDOEL, V. P. A. $M$. An autopsy of epiderniologic methods: the case of "poppers" in the early epidemic of the acquired immunodeficiency syndrome (AIDS). [Reviews and Commentary.] $A m, J$. Epidemiol, 129(3), 1989.
27. WING, S. Concepts in modern epidemiology: population, risk, dose response and confounding. Salvador, 1993. [Texto apresentado na Oficina de Trabalho "Teoria critica da epidemiologia" do Departamento de Medicina Preventiva da UFBA, Salvador, 1993].

\begin{abstract}
The foundations on which the concept of risk has been constructed are discussed. A description of Rubin's model of causal inference, which was first developed in the domain of applled statistics, and later incorporated into a branch of epidemiology, is taken as the starting point. Analysis of the premisses of causal inference brings to light the logical stages in the construction of the concept of risk, allowing it to be understood "from the inside". The abovementioned branch of statistics and epidemiology seeks to demonstrate that statistics can infer causality instead of simply revealing statistical associations; the model gives the basis for estimating that which way be defined as the effect of a cause. Using this procedural distinction between causal inference and association, the model also seeks to difierentiate between the epidemiologial dimension of concepts and the merely statiscal dimension. This leads to greater complexity when handing the concepts of interation and coofounding. The redective aspects inherent in this methodological construction of risk are here high lighted. Thus, whether applied to individual or populational inferences, this methodological construction imposes limits that need to be taken into account in its theoretical and pratical application to epidemiology.
\end{abstract}

Risk. Inference. Causality. Proportional hazards models. 